\title{
A novel manganese complex selectively induces malignant glioma cell death by targeting mitochondria
}

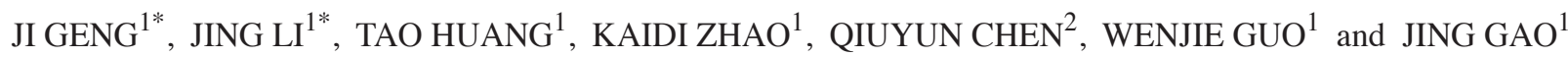 \\ ${ }^{1}$ Department of Pharmacy, School of Pharmacy; ${ }^{2}$ Department of Applied Chemistry, \\ School of Chemistry and Chemical Engineering, Jiangsu University, Zhenjiang, Jiangsu 212013, P.R. China
}

Received May 23, 2015; Accepted April 14, 2016

DOI: $10.3892 / \mathrm{mmr} .2016 .5509$

\begin{abstract}
Despite advances in treatment, malignant glioma commonly exhibits recurrence, subsequently leading to a poor prognosis. As manganese $(\mathrm{Mn})$ compounds can be transported by the transferrin-transferrin receptor system, the present study synthesized and examined the potential use of Adpa-Mn as a novel antitumor agent. Adpa-Mn time and dose-dependently inhibited U251 and C6 cell proliferation; however, it had little effect on normal astrocytes. Apoptosis was significantly elevated following treatment with Adpa-Mn, as detected by chromatin condensation, Annexin V/propidium iodide staining, cytochrome $c$ release from mitochondria to the cytoplasm, and the activation of caspases-9, -7 and -3 and poly (ADP-ribose) polymerase. In addition, Adpa-Mn enhanced fluorescence intensity of monodansylcadaverine and elevated the expression levels of the autophagy-related protein microtubule-associated protein 1 light chain 3. Pretreatment with the autophagy inhibitors 3-methyladenine and chloroquine enhanced Adpa-Mn-induced cell inhibition, thus indicating that autophagy has an essential role in this process. Furthermore, evidence of mitochondrial dysfunction was detected in the Adpa-Mn-treated group, including disrupted membrane potential, elevated levels of reactive oxygen species (ROS) and depleted adenosine triphosphate. Conversely, treatment with the mitochondrial permeability transition inhibitor cyclosporin A reversed Adpa-Mn-induced ROS production, mitochondrial damage and cell apoptosis, thus suggesting that Adpa-Mn may target the mitochondria. Taken together, these data suggested that Adpa-Mn may be considered for use as a novel anti-glioma therapeutic option.
\end{abstract}

Correspondence to: Professor Jing Gao or Dr Wenjie Guo, Department of Pharmacy, School of Pharmacy, Jiangsu University, 301 Xuefu Road, Zhenjiang, Jiangsu 212013, P.R. China

E-mail: jinggao@ujs.edu.cn

E-mail: guowj06@126.com

${ }^{*}$ Contributed equally

Key words: manganese complex, glioma, apoptosis, autophagy, mitochondria

\section{Introduction}

Malignant glioma, which is the most common type of primary brain tumor, is highly aggressive and metastatic. Survival ranges between 12 and 40 months from initial diagnosis, and between 6 and 18 months following recurrence, despite treatment with standard therapies, including surgery, chemotherapy and radiotherapy (1-3). Therefore, it is necessary to develop novel strategies for the treatment of glioma. Mitochondria are unique cellular organelles, the primary role of which is to generate adenosine triphosphate (ATP) through oxidative phosphorylation, in order to ensure survival (4). Previous studies have reported that mitochondria have a central role in regulating proliferation, apoptosis and autophagy in cancer cells (5-7). Therefore, disturbing mitochondrial metabolism or interfering with mitochondrial membrane permeabilization may be considered a promising therapeutic approach to cancer.

Autophagy is a lysosomal degradation pathway by which cells consume intracellular materials, including damaged organelles, proteins or hazardous substances, in order to maintain cellular homeostasis (8). Autophagy is upregulated in response to cellular stress, including starvation, mitochondrial stress, endoplasmic reticulum stress and pathogenic infection (9). The emerging role of autophagy in cancer is considered to be a double-edged sword. Autophagy has been reported to render cancer cells able to tolerate therapy-induced stress; however, autophagy can also digest organelles and limit tumorigenesis (10). Therefore, modulating the autophagic pathway may provide novel approaches to cancer therapy and prevention.

Manganese (Mn) is an essential mineral that can influence intracellular and extracellular metabolism associated with the mitochondria (11). Apoptosis is activated by Mn compounds, which induce the rupture of DNA and the release of cytochrome $c$ from the mitochondrial intermembrane space to the cytosol (12). In addition, several enzymes have been identified, which naturally contain $\mathrm{Mn}$, including pyruvate carboxylase, arginase and $\mathrm{Mn}$ superoxide dismutase $(13,14)$. The main purpose of $\mathrm{Mn}$ is as a coactivator of superoxide dismutase in mitochondria (15). It has been reported that Mn (II) ions are predominantly transported by divalent metal transporter 1 (DMT-1) and the transferrin-transferrin receptor (Tf-TfR) system, which is highly expressed in various tumors (16). It has previously been demonstrated that Mn (II) ions can 
transport across the rat blood-brain barrier, through saturable and Tf-dependent transport mechanisms, to the brain (17-19). Based on the aforementioned findings, the present study hypothesized that Mn-containing compounds may cross the blood-brain barrier and selectively kill glioma cells via DMT-1 or TfR.

The present study indicated that the complex [(Adpa) $\left.\mathrm{Mn}(\mathrm{Cl})\left(\mathrm{H}_{2} \mathrm{O}\right)\right]$, designated as Adpa-Mn, which is an inorganic compound comprised of Adpa [bis(2-pyridylmethyl) amino-2-propionic acid] and $\mathrm{Mn}$, is a promising novel anti-glioma agent with potent selective activity in vitro. Furthermore, it was verified that Adpa-Mn induces apoptotic cell death and cytoprotective autophagy by triggering mitochondrial dysfunction.

\section{Materials and methods}

Materials. The compound Adpa-Mn was synthesized by Professor Chen Qiuyun (Fig. 1A) as previously described (20). Cisplatin was purchased from Jiangsu Hengrui Medicine Co., Ltd.(Lianyungang, China). 3-[4,5-dimehyl-2-thiazolyl]-2,5-diphenyl-2H-tetrazolium bromide (MTT) was purchased from Amresco, LLC (Solon, OH, USA). JC-1 for mitochondrial membrane potential, Dulbecco's modified Eagle's medium (DMEM) for U251 and C6, RPMI-1640 for rat astrocyte cells, trypsin-EDTA solution for cell detachment and the Annexin V/propidium iodide (PI) kit for apoptotic detection were purchased from Thermo Fisher Scientific, Inc. (Waltham, MA, USA). Ferric citrate, 4',6-diamidino-2-phenylindole (DAPI), desferrioxamine (DFO) and cyclosporin A (CsA) were purchased from Sigma-Aldrich (St. Louis, MO, USA). Fetal bovine serum (FBS) was obtained from Sijiqing Biological Engineering Materials Co., Ltd. (Hangzhou, China). The 2',7'-dichlorofluorescein-diacetate (DCFH-DA) kit was purchased from Beyotime Institute of Biotechnology (Nantong, China). Anti-microtubule-associated protein 1 light chain 3 (LC3; cat. no. 3868; 1:1,000), anti-poly (ADP-ribose) polymerase (PARP; cat. no. 9536; 1:1000), anti-caspase-9 (cat. no. 9508, 1:1,000), anti-caspase-7 (cat. no. 9494, 1:1,000), anti-caspase-3 (cat. no. 9665; 1:1,000) and anti-B-cell lymphoma 2 (Bcl-2;cat. no. 15071; 1:1,000), cytochrome $c$ (cat. no. 4280, 1:1000), cytochrome $c$ oxidase subunit IV (cat. no. 4850; 1:1,000) were purchased from Cell Signaling Technology, Inc. (Danvers, MA, USA) and were diluted with $1 \%$ bovine serum albumin (BSA). Anti- $\beta$-actin antibody (cat. no. SC-8432; 1:200) was obtained from Santa Cruz Biotechnology, Inc. (Dallas, TX, USA), anti-DMT-1 antibody (cat. no. 20507-1-AP; 1:200) was purchased from Proteintech Group, Inc. (Rosemont, IL, USA), and anti-TfR antibody (cat. no. MA5-11441; 1:200) was purchased from Thermo Fisher Scientific, Inc. Monodansylcadaverin (MDC), 3-methyladenine (3-MA), N-acetylcysteine (NAC), ferric citrate, DFO, CsA and chloroquine (CQ) were purchased from Sigma-Aldrich. All other chemicals were of high purity, and were purchased from commercial sources. The study was approved by the ethics committee of Jiangsu University (Zhenjiang, China).

Cell culture. The U251 human glioma cell line, and the C6 rat glioma cell line were obtained from the Cancer Cell
Repository (Shanghai Cell Bank; Shanghai, China). The cells were maintained in DMEM supplemented with $10 \%(\mathrm{v} / \mathrm{v})$ heat-inactivated FBS and antibiotics $(100 \mathrm{U} / \mathrm{ml}$ penicillin and $100 \mathrm{U} / \mathrm{ml}$ streptomycin; Beyotime Institute of Biotechnology) at $37^{\circ} \mathrm{C}$ in a humidified atmosphere containing $5 \% \mathrm{CO}_{2}$. Primary astrocyte cells isolated from three newborn Sprague-Dawley rats were cultured in RPMI-1640 as described previously (21).

Cell viability assay. The cells were plated at a density of $\sim 4 \times 10^{3}$ viable cells/well in 96-well plates in the presence of $5,10,20,30$, or $40 \mu \mathrm{M}$ of the compound for 12,24 , or $36 \mathrm{~h}$. MTT $(1 \mathrm{mg} / \mathrm{ml})$ was added for $3 \mathrm{~h}$ followed by dimethyl sulfoxide to dissolve the formazan product, and the U251 and C6 cell viabilities were measured using a 96-well plate reader (SpectraMax 190; Molecular Devices,Sunnyvale, CA, USA) at $490 \mathrm{~nm}$.

Cell apoptosis assay. The cells were plated at a density of $\sim 1 \times 10^{5}$ viable cells/well in 6-well plates. Cells were harvested following 5, 10, $20 \mu \mathrm{M}$ Adpa-Mn treatments for $24 \mathrm{~h}$. Cells were collected using trypsin-EDTA and incubated with Annexin V/PI at room temperature for $15 \mathrm{~min}$ in the dark, then analyzed using a FACSCalibur flow cytometer (BD Biosciences, Franklin Lakes, NJ, USA). CellQuest (Becton Dickinson, Mountain View, CA, USA) was applied to analyze flow cytometric results. Annexin $\mathrm{V}^{+} / \mathrm{PI}^{-}$and Annexin $\mathrm{V}^{+} / \mathrm{PI}^{+}$ cells were considered to be early and late phase apoptotic cells, respectively.

Visualization of MDC-labeled vacuoles. Autophagic vacuoles were labeled with MDC. Briefly, U251 cells grown on coverslips were incubated with $50 \mu \mathrm{M}$ MDC in phosphate-buffered saline (PBS) at $37^{\circ} \mathrm{C}$ for $10 \mathrm{~min}$. Alterations in cellular fluorescence were observed under a Nikon eclipse Ti fluorescence microscope (Nikon Corporation, Tokyo, Japan; excitation, 380-420 nm; emission, $450 \mathrm{~nm}$ ).

Western blot analysis. Proteins were extracted from the cells using lysis buffer (30 mM Tris, $\mathrm{pH} \mathrm{7.5;150} \mathrm{mM} \mathrm{sodium}$ chloride; $1 \mathrm{mM}$ phenylmethylsulfonyl fluoride; $1 \mathrm{mM}$ sodium orthovanadate; $1 \%$ Nonidet P-40; $10 \%$ glycerol; and phosphatase and protease inhibitors; Roche Diagnostics, Basel, Switzerland). Then, $20 \mu \mathrm{g}$ protein was separated by $15 \%$ sodium dodecyl sulfate-polyacrylamide gel electrophoresis at a constant voltage setting of $110 \mathrm{~V}$ for $80 \mathrm{~min}$ and were electrophoretically transferred onto polyvinylidene fluoride membranes (Thermo Fisher Scientific Inc.) at a constant current setting of $300 \mathrm{~mA}$ for $90 \mathrm{~min}$. Membrane blocking was performed with $1 \%$ BSA for $1 \mathrm{~h}$. The membranes were probed with primary antibodies diluted with $1 \% \mathrm{BSA}$ overnight at $4^{\circ} \mathrm{C}$, followed by an incubation with horseradish peroxidase-conjugated goat anti-mouse (cat. no. sc-2969) and goat anti-rabbit (cat. no. sc-2768; Santa Cruz Biotechnology, Inc.) secondary antibodies for $2 \mathrm{~h}$ at room temperature. Detection was performed using a LumiGLO Chemiluminescent Substrate system (KPL, Gaithersburg, MD, USA).

Mitochondrial membrane potential assay. Alterations to mitochondrial membrane potential were measured using JC-1. U251 cells were washed with PBS and were incubated with 

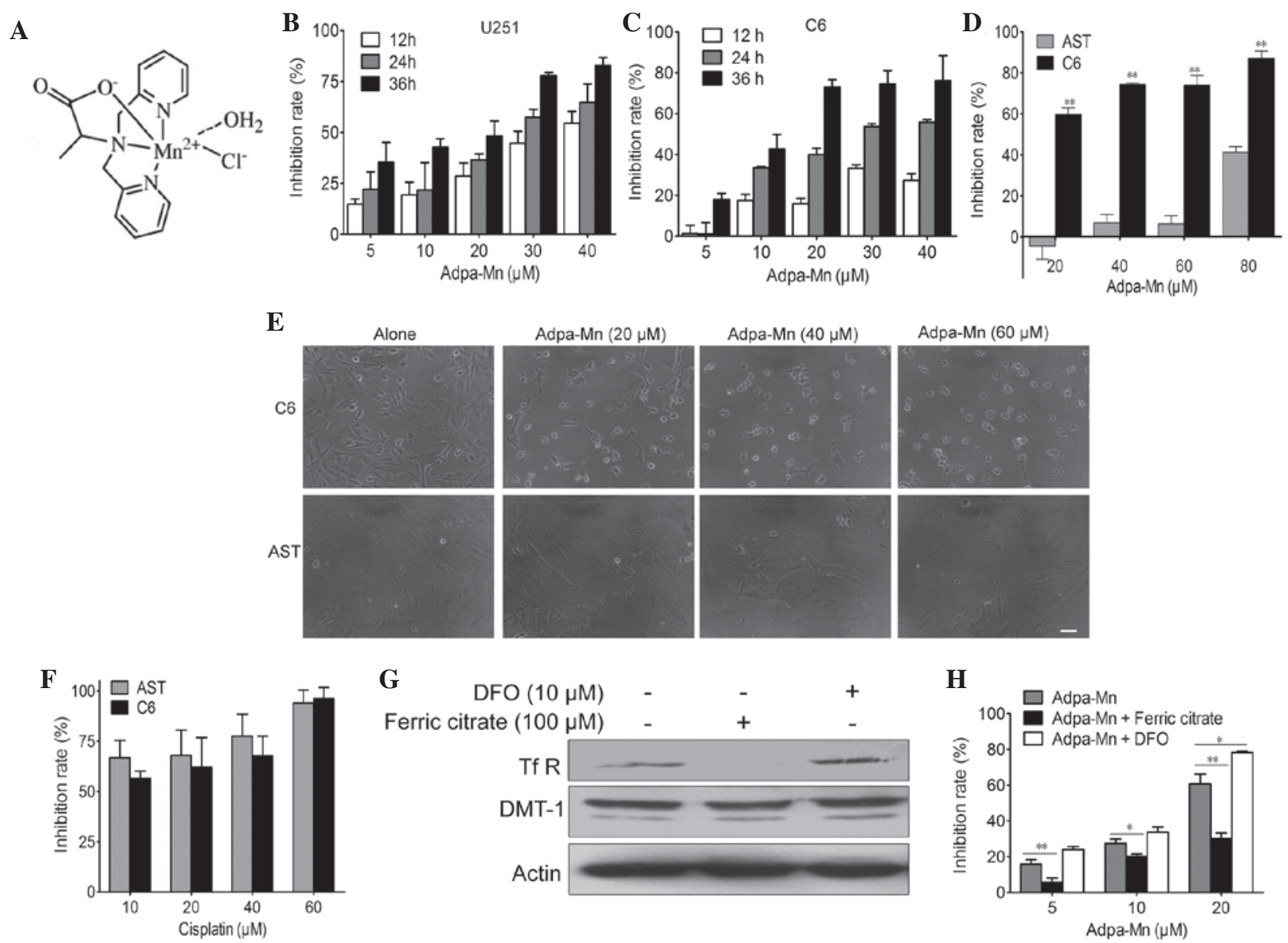

Figure 1. Adpa-manganese (Mn) exhibited selective inhibition on glioma cell proliferation. (A) Structure of Adpa-Mn. (B and C) U251 and C6 cells were exposed to various concentrations of Adpa-Mn, and cell proliferation was determined by 3-[4,5-dimehyl-2-thiazolyl]-2,5-diphenyl-2H-tetrazolium bro mide (MTT) assay every $12 \mathrm{~h}$. (D) C6 cells and rat astrocyte cells (AST) were exposed to various concentrations of Adpa-Mn for $24 \mathrm{~h}$, and cell proliferation was determined by MTT assay. (E) Images of cellular morphological alterations were captured (scale bar, $10 \mu \mathrm{m}$ ). (F) C6 cells and AST cells were exposed to various concentrations of cisplatin for $24 \mathrm{~h}$, and cell proliferation was determined by MTT assay. (G) Expression of divalent metal transporter 1 (DMT-1) and transferrin receptor (TfR) in U251 cells following ferric citrate $(100 \mu \mathrm{M})$ or desferrioxamine (DFO; $10 \mu \mathrm{M})$ pretreatment for $24 \mathrm{~h}$, as determined by western blotting. (H) U251 cells were pretreated with ferric citrate $(100 \mu \mathrm{M})$ or DFO $(10 \mu \mathrm{M})$ for $24 \mathrm{~h}$, and were then treated with Adpa-Mn for $24 \mathrm{~h}$. Cell proliferation was determined by MTT assay. Data are presented as the mean \pm standard deviation of three independent experiments. ${ }^{*} \mathrm{P}<0.05,{ }^{* * *} \mathrm{P}<0.01$.

$5 \mu \mathrm{g} / \mathrm{ml} \mathrm{JC}-1$ at $37^{\circ} \mathrm{C}$ for $30 \mathrm{~min}$. Cells were then washed twice with PBS and were immediately assessed by fluorescence spectrometry (Spectra Max Gemini; Molecular Devices). A $488-\mathrm{nm}$ filter was used to detect the excitation of JC-1. Emission filters of 535 and $595 \mathrm{~nm}$ were used to quantify the population of mitochondria exhibiting green (JC-1 monomers) and red (JC-1 aggregates) fluorescence. The ratio of red/green fluorescence was used to reflect the mitochondrial membrane potential.

Determination of intracellular ATP levels. ATP content was measured according to the luciferin-luciferase method, which is based on the requirement of ATP for luciferase to produce light. The cells were plated at a density of $\sim 1 \times 10^{5}$ viable cells/well in 6-well plates. Cells were harvested following treatment with $20 \mu \mathrm{M}$ Adpa-Mn for 6,12 and $24 \mathrm{~h}$ and were assayed for ATP using a chemical luciferase ATP assay kit (Beyotime Institute of Biotechnology). The quantity of ATP in the experimental samples was calculated from a standard curve prepared with ATP, and was expressed as $\mathrm{nmol} / \mathrm{mg}$ protein.
Statistical analysis. Differences between groups were analyzed using a two-tailed Student's t-test with GraphPad Prism version 5.00 for Windows (GraphPad Software, San Diego CA, USA) and differences among groups were analyzed using one-way analysis of variance. All values are expressed as the mean \pm standard deviation and $\mathrm{P}<0.05$ was considered to indicate a statistically significant difference. All experiments were repeated at least three times.

\section{Results}

Adpa-Mn exhibits significant and selective anti-glioma activity. To determine whether Adpa-Mn has an inhibitory effect on glioma cells, the U251 human glioma cell line and the $\mathrm{C} 6$ rat glioma cell line were subjected to an MTT assay. Treatment with Adpa-Mn inhibited U251 and C6 cell proliferation in a dose- and time-dependent manner (Fig. 1B and 1C).

Subsequently, it was determined whether Adpa-Mn exhibits cancer cell selectivity. Treatment with Adpa-Mn exhibited significant selectivity towards glioma cells (C6) over normal rat astrocytes (Fig. 1D and E), whereas cisplatin did not exert 
A Alone

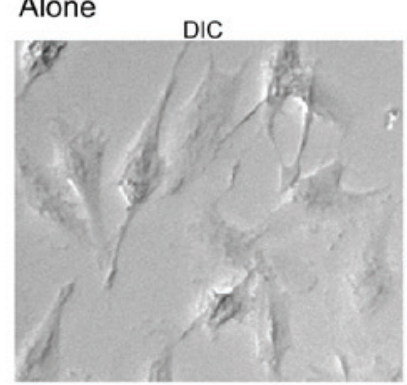

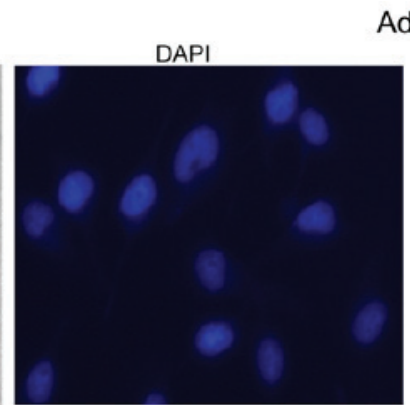

Adpa-Mn $(20 \mu \mathrm{M})$
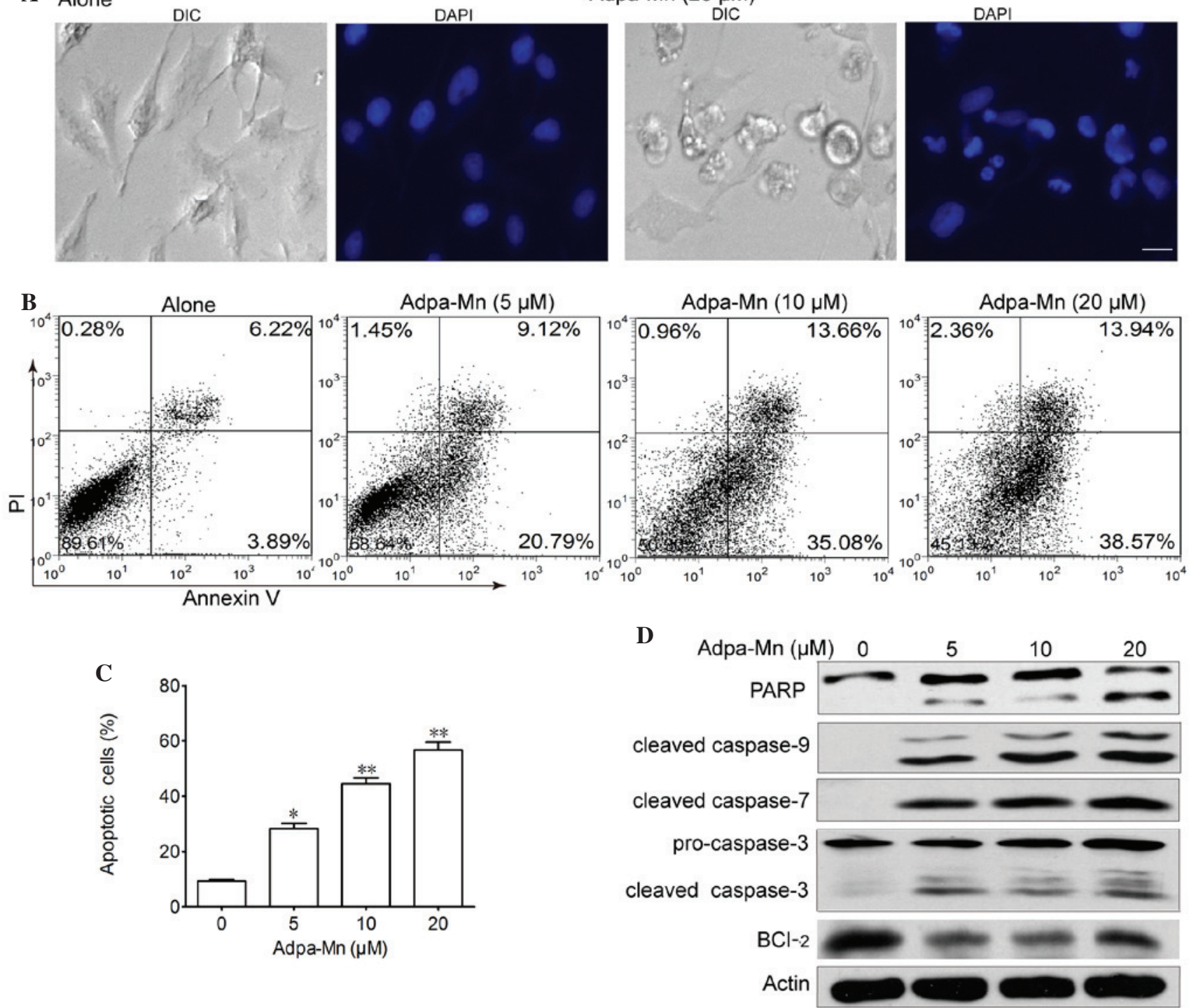

$\mathbf{E}$
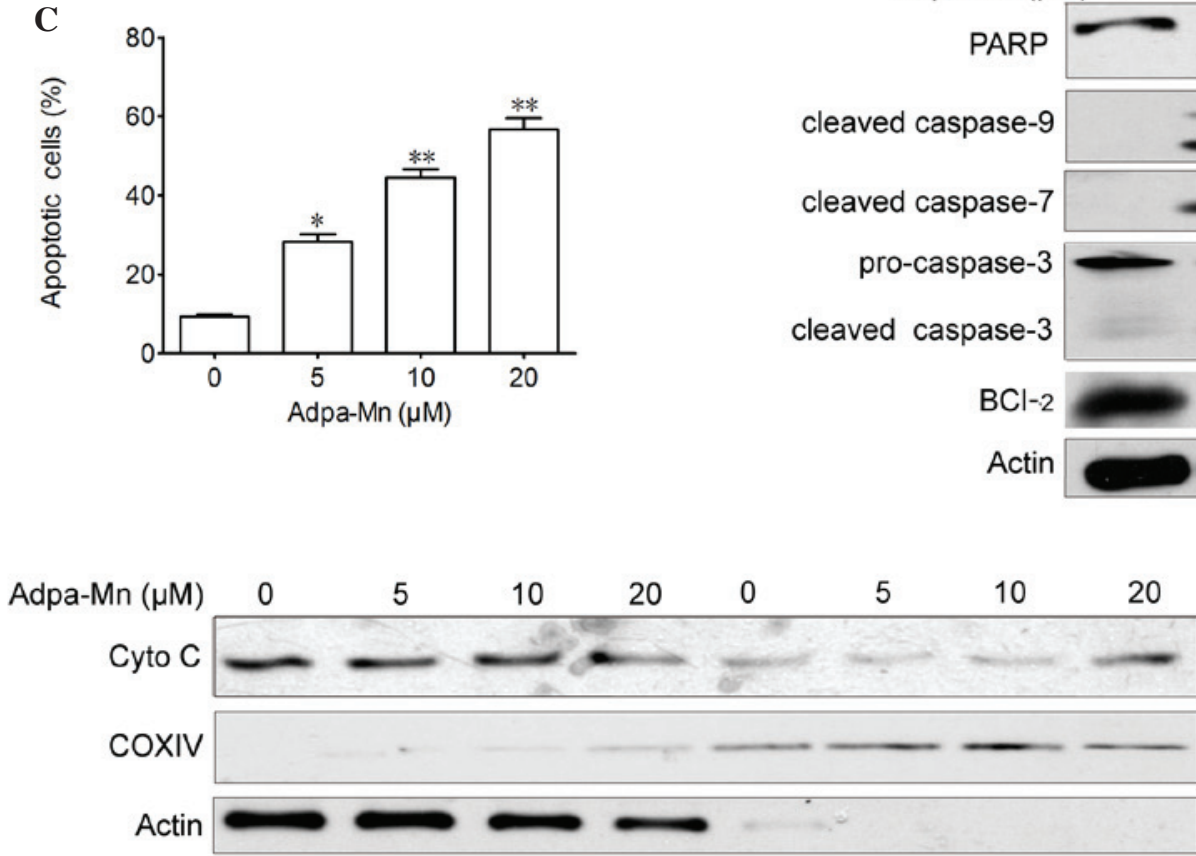

Figure 2. Adpa-manganese (Mn) induced apoptotic cell death via the mitochondrial pathway. (A) U251 cells were treated with Adpa-Mn (20 $\mu \mathrm{M})$ for $24 \mathrm{~h}$. The cells were stained with 4',6-diamidino-2-phenylindole (DAPI) and images of nuclear morphology were captured. Scale bar, $5 \mu \mathrm{m}$. (B and C) U251 cells treated with Adpa-Mn were collected and subjected to Annexin V/propidium iodide (PI) analysis. (D) Poly (ADP-ribose) polymerase (PARP) and caspase activation were determined by western blotting. (E) Release of cytochrome $c$ (Cyto C) from the mitochondria was detected by western blotting. COXIV served as a mitochondrial control and actin served as a cytoplasmic control. Data are presented as the mean \pm standard deviation of three independent experiments. ${ }^{*} \mathrm{P}<0.05,{ }^{* *} \mathrm{P}<0.01$ vs. $0 \mu \mathrm{M}$ Adpa-Mn. Bcl-2, B-cell lymphoma 2; COXIV, cytochrome c oxidase subunit IV; DIC, differential interference contrast.

this selectivity (Fig. 1F). This preferential toxicity toward cancer cells over non-cancer cells suggests the potential use of this compound as an antitumor therapeutic option.

Due to the high expression of TfR on tumor cells, the cancer cell selectivity of Adpa-Mn may be due to its transport mechanism. As detected by western blotting and MTT assay, when the expression of TfR was inhibited by ferric citrate $(100 \mu \mathrm{M})$ pretreatment for $24 \mathrm{~h}$, the inhibitory effect of Adpa-Mn on
U251 cells was significantly decreased. Conversely, when TfR expression was upregulated following DFO $(10 \mu \mathrm{M})$ pretreatment for $24 \mathrm{~h}$, Adpa-Mn induced U251 cell inhibition more efficiently (Fig. 1G and $\mathrm{H}$ ).

Adpa-Mn induces apoptotic cell death. To investigate the potential anticancer mechanisms of Adpa-Mn, U251 malignant glioma cells were treated with various concentrations of 


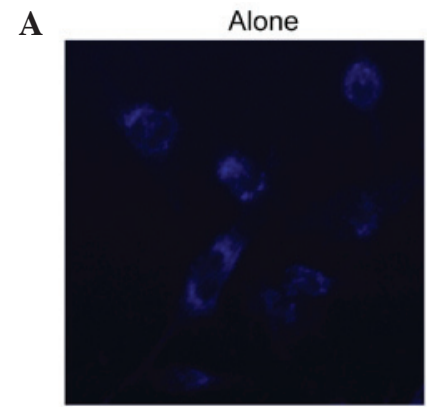

B
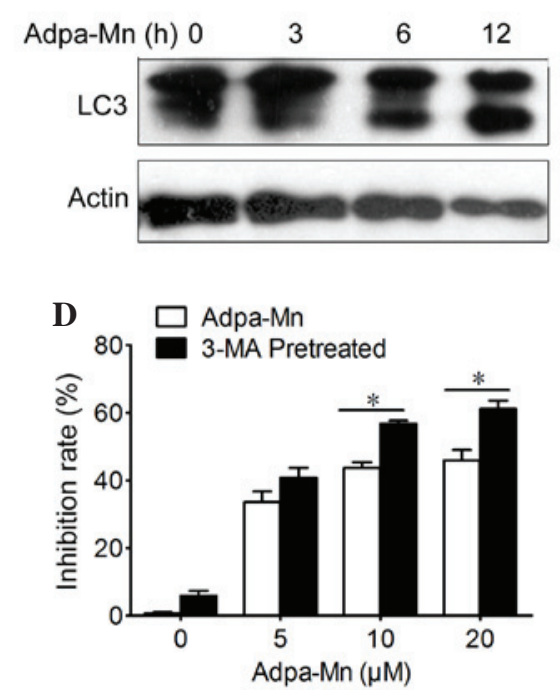

Adpa-Mn $(10 \mu \mathrm{M})$

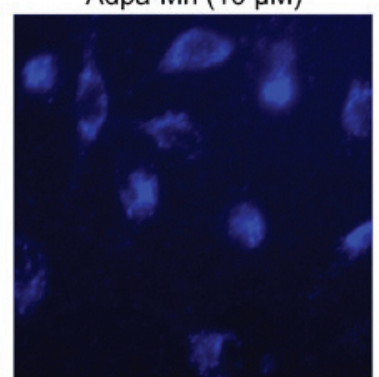

C Adpa-Mn $(\mu \mathrm{M}) 0 \quad 10 \quad 20 \quad 3-M A+20$

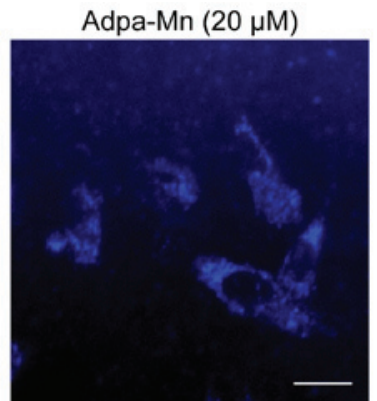

LC3

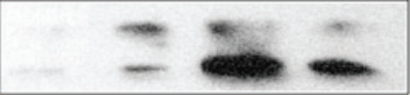

Actin
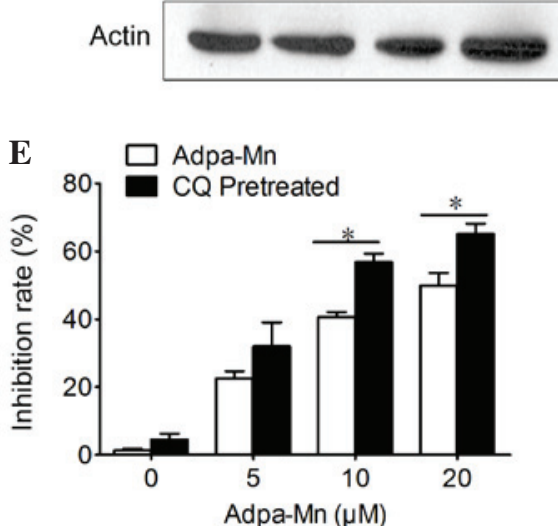

Figure 3. Adpa-manganese (Mn) induced cell protective autophagy. (A) U251 cells were treated with Adpa-Mn for $12 \mathrm{~h}$ and were then incubated with $0.05 \mathrm{mM}$ monodansylcadaverine for $10 \mathrm{~min}$. Cells were analyzed by fluorescence microscopy. Scale bar, $5 \mu \mathrm{m}$. (B and C) Protein expression levels of microtubule-associated protein 1 light chain 3 (LC3) was analyzed by western blotting. (D and E) U251 cells were treated with $20 \mu \mathrm{M}$ Adpa-Mn for $24 \mathrm{~h}$ with or without 3-methyladenine (3-MA) or chloroquine (CQ) pretreatment (2 h). 3-[4,5-dimehyl-2-thiazolyl]-2,5-diphenyl-2H-tetrazolium bro mide assay was used to evaluate cell viability. Data are presented as the mean \pm standard deviation of three independent experiments. ${ }^{*} \mathrm{P}<0.05$.

Adpa-Mn for 24 h. Apoptotic cell death was detected using 4',6-diamidino-2-phenylindole, a typical nuclear stain that can visualize nuclear condensation. The results demonstrated that nuclei became condensed and fragmented, and apoptotic bodies were detected following treatment with Adpa-Mn (Fig. 2A). Adpa-Mn-induced apoptosis was further detected using Annexin V/PI staining. As presented in Fig. 2B and C, Annexin $\mathrm{V}^{+}$cells were abundantly increased from 10.1 to 48.7 and $52.5 \%$ following treatment with 10 and $20 \mu \mathrm{M}$ Adpa-Mn, respectively. To determine the involvement of the caspase cascade in Adpa-Mn-induced apoptosis, the expression levels of caspases were detected by western blotting. The hallmarks of apoptosis, including PARP, caspase-9, caspase-7 and caspase-3, were activated/cleaved in U251 cells following treatment with Adpa-Mn in a dose-dependent manner (Fig. 2D). Consistent with the decreased expression of Bcl-2 (Fig. 2D), which is an integral membrane protein located mainly on the outer mitochondrial membrane that inhibits apoptosis, cytochrome $c$ was transferred from the mitochondrial intermembrane space to the cytoplasm (Fig. 2E). These results suggest that Adpa-Mn may induce significant apoptosis of U251 cells.

Adpa-Mn induces protective autophagy. Cellular features of necrosis, apoptosis and autophagy frequently occur together in response to death signals and toxic stress (22). The present study determined whether Adpa-Mn was able to induce cell death via autophagy. Autophagy is characterized by the accumulation of vesicles and the formation of autophagosomes, which can be detected by the presence of membrane-bound LC3-phospholipid conjugates (23). MDC is a specific marker of autophagic vacuoles (Fig. 3A) and treatment with Adpa-Mn $(10$ and $20 \mu \mathrm{M})$ for $12 \mathrm{~h}$ triggered the accumulation of MDC-stained acidic vesicular organelles (AVO) in U251 cells. In addition, Adpa-Mn yielded a time- and dose-dependent increase in the expression levels of LC3 II (the processed form of LC3; Fig. 3B and C). In addition, the LC3 II/LC3 I ratio was increased in a dose- and time-dependent manner following treatment with Adpa-Mn. These data suggest that autophagy was enhanced following treatment with Adpa-Mn. It has previously been reported that apoptosis and autophagy are not mutually exclusive pathways, but have been shown to act in synergy and also to counter each other (24). Pretreatment with 3-MA, an inhibitor of autophagy, and CQ, an inhibitor of lysosomal degradation, decreased Adpa-Mn induced LC3 II accumulation and LC3 II/LC3 I ratio (Fig. 3C), and accelerated cell death (Fig. 3D and E). Autophagy inhibition decreased cell viability, which indicated that Adpa-Mn-induced autophagy exerts a protective role in U251 cells. 

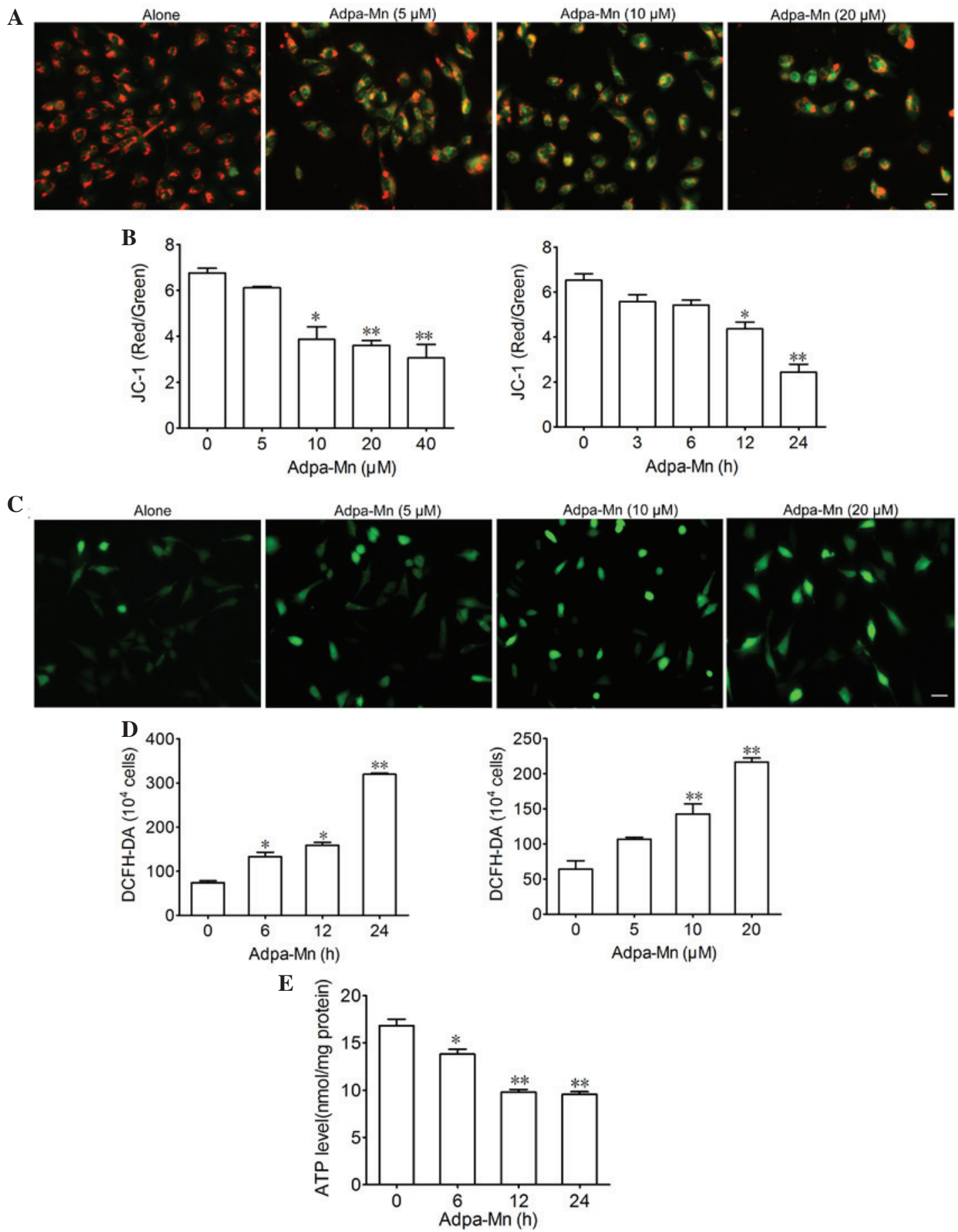

Figure 4. Adpa-manganese (Mn) treatment induced mitochondrial dysfunction. (A and B) U251 cells were exposed to Adpa-Mn (20 $\mu \mathrm{M}$ for 3, 6, 12 and 24 h; or $5,10,20$ and $40 \mu \mathrm{M}$ for $12 \mathrm{~h}$ ) and mitochondrial membrane potential was determined by JC-1 staining and fluorescence microscopy (scale bar, $10 \mu \mathrm{m}$ ), and was quantified by fluorescence spectrometry (red/green fluorescence ratio). (C and D) U251 cells were exposed to Adpa-Mn (20 $\mu \mathrm{M}$ for 6 , 12 and 24 h; or 5,10 and $20 \mu \mathrm{M}$ for $12 \mathrm{~h}$ ) and reactive oxygen species generation was detected by 2',7'-dichlorofluorescein-diacetate (DCFH-DA) staining (scale bar, $10 \mu \mathrm{m}$ ). (E) U251 cells were exposed to $20 \mu \mathrm{M}$ Adpa-Mn for 6, 12 and $24 \mathrm{~h}$, and adenosine triphosphate (ATP) levels were measured using the luciferin-luciferase assay. Data are presented as the mean \pm standard deviation of three independent experiments. ${ }^{*} \mathrm{P}<0.05,{ }^{* *} \mathrm{P}<0.01$ vs. control $(0 \mu \mathrm{M} / 0 \mathrm{~h}$ Adpa-Mn).

Adpa-Mn triggers mitochondrial dysfunction. Since mitochondria are critical in apoptosis and autophagy, the present study aimed to determine the effects of Adpa-Mn on mitochondrial function. JC-1 was used to evaluate mitochondrial membrane potential. Treatment with 5, 10 and $20 \mu \mathrm{M}$ Adpa-Mn for $12 \mathrm{~h}$ led to a decrease in mitochondrial membrane potential, as detected by enhanced green intensity and reduced red intensity of JC-1 (Fig. 4A). Subsequently, the ratio of red and green fluorescence intensity was determined using a fluorescent microplate reader, which indicated a dose- and time-dependent decrease (Fig. 4B). DCFH-DA was used to determine the effects of Adpa-Mn on intracellular ROS generation in U251 cells. As presented in Fig. 4C and D, the fluorescence intensity of DCFH-DA per $1 \times 10^{4}$ cells was significantly elevated following treatment with Adpa-Mn in a dose- and time-dependent manner, thus suggesting that 

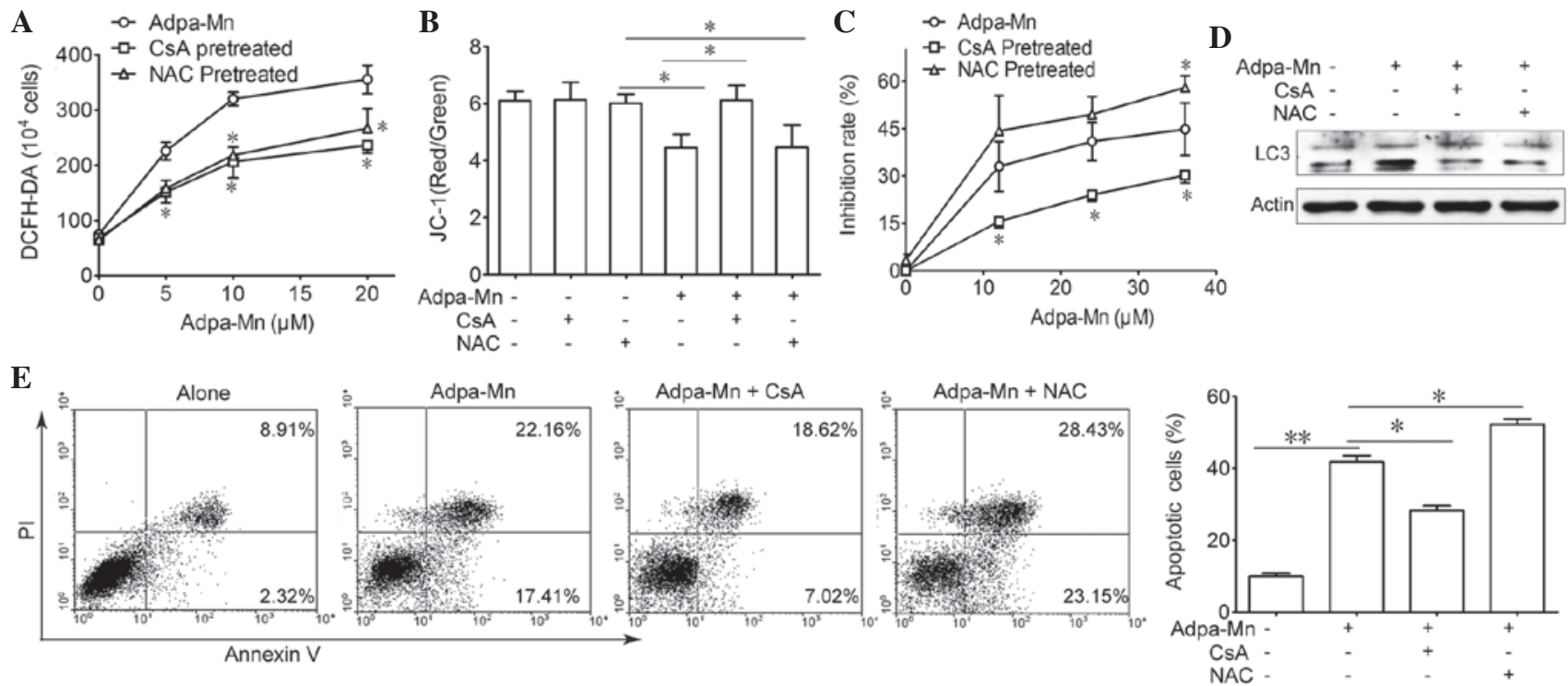

Figure 5. Cell death induced by Adpa-manganese (Mn) treatment was dependent on mitochondrial dysfunction. (A) U251 cells were exposed to $5,10,15$ and $20 \mu \mathrm{M}$ Adpa-Mn for $24 \mathrm{~h}$ with or without $\mathrm{N}$-acetylcysteine (NAC; $5 \mathrm{mM}$ ) or cyclosporin A (CsA; $2 \mu \mathrm{M}$ ) pretreatment $(2 \mathrm{~h})$. Reactive oxygen species (ROS) levels were examined by 2',7'-dichlorofluorescein-diacetate(DCFH-DA) staining. (B) U251 cells were exposed to $20 \mu \mathrm{M}$ Adpa-Mn for $12 \mathrm{~h}$ with or without NAC (5 mM) or CsA $(2 \mu \mathrm{M})$ pretreatment $(2 \mathrm{~h})$, and mitochondrial membrane potential was evaluated by JC-1 staining. (C) U251 cells were exposed to 10,20,30 and 40 $\mu \mathrm{M}$ Adpa-Mn for $24 \mathrm{~h}$ with or without NAC $(5 \mathrm{mM})$ orCsA $(2 \mu \mathrm{M})$ pretreatment $(2 \mathrm{~h})$, and cell viability was examined by 3-[4,5-dimehyl-2-thiazolyl]-2,5-diphenyl-2H-tetrazoliumbro mide assay. (D) U251 cells were exposed to $20 \mu \mathrm{M}$ Adpa-Mn for $12 \mathrm{~h}$ with or without NAC (5 mM) or CsA ( $2 \mu \mathrm{M})(2 \mathrm{~h})$, and microtubule-associated protein 1 light chain 3 (LC3) expression was evaluated by western blotting. (E) U251 cells were exposed to $20 \mu \mathrm{M}$ Adpa-Mn for $24 \mathrm{~h}$ with or without NAC (5 mM) or CsA $(2 \mu \mathrm{M})$ pretreatment $(2 \mathrm{~h})$, and apoptosis was evaluated by Annexin V/propidium iodide (PI) staining. Data are presented as the mean \pm standard deviation of three independent experiments. ${ }^{*} \mathrm{P}<0.05$ and ${ }^{* *} \mathrm{P}<0.01$ vs. Adpa-Mn.

intracellular ROS production was increased. Furthermore, exposure of U251 cells to Adpa-Mn resulted in ATP depletion (Fig. 4E). These results suggest that Adpa-Mn triggers mitochondrial dysfunction in U251 cells.

Adpa-Mn induces cell death dependent on mitochondrial dysfunction. Due to the finding that Adpa-Mn triggers mitochondrial membrane potential breakdown and ROS generation, the present study aimed to elucidate the association between ROS, mitochondria and Adpa-Mn-induced cell death (Fig. 5). Pretreatment with CsA (2 $\mu \mathrm{M})$, an inhibitor of the mitochondrial permeability transition pore (MPTP) and NAC, a ROS scavenger, reduced Adpa-Mn-induced ROS production (Fig. 5A), whereas only CsA pretreatment was able to reverse Adpa-Mn-induced membrane potential collapse (Fig. 5B). Furthermore, MTT assay and Annexin V/PI staining indicated that cell death or apoptosis triggered by Adpa-Mn was significantly reduced by CsA pretreatment, but was aggravated to some extent by NAC pretreatment (Fig. 5C and E). In addition, CsA and NAC were able to reduce LC3 II expression (Fig. 5D). These findings indicate that Adpa-Mn may disrupt the mitochondrial membrane to induce apoptosis and protective autophagy in U251 cells.

\section{Discussion}

The success of cisplatin in the treatment of patients with cancer has resulted in the hypothesis that other metal complexes may be considered as potential drugs in future chemotherapy regimens. The present study aimed to verify whether the designed Mn-compound, Adpa-Mn, may be used as an anti-glioma lead compound. In the present study, Adpa-Mn was demonstrated to be active against glioma cells, whereas it had little effect on normal astrocytes. In addition, Adpa-Mn time and dose-dependently induced mitochondrial dysfunction, as determined by mitochondrial collapse and ROS generation, finally resulting in apoptotic cell death.

Apoptosis is a type of cell death that is characterized by nuclear condensation and fragmentation, and apoptotic body emergence without plasma membrane breakdown. During apoptosis, numerous death signals converge on mitochondria, particularly the release of cytochrome $c$ as a consequence of the increased permeability of the outer mitochondrial membrane, which subsequently activates downstream caspase signaling (25). In the present study, Adpa-Mn was revealed to induce apoptosis mediated by the mitochondrial pathway (Fig. 2).

Autophagy is a dynamic process, which is often termed autophagic flux, whereby autophagosomes are formed in response to stimuli, engulf the cellular content and damaged organelles, and fuse with lysosomes; the contents of the autophagosome are subsequently degraded (26). Autophagy has previously been referred to as a physiological process that has a protective role in cells that encounter environmental stress, including starvation and pathogenic infection (27). Excess autophagy can also act as a pro-death mechanism; therefore, it has been classified as type II programmed cell death or autophagic cell death (28). In U251 cells, the following characteristics of autophagy were detected following Adpa-Mn treatment: Formation of AVOs and elevated ratio of LC3-II to LC3-I. When combined with the autophagy inhibitors, CQ and 3-MA, cell viability was markedly inhibited (Fig. 3). These results suggested that autophagy may have a protective role during Adpa-Mn 
treatment, which is similar to what has been reported for other chemicals $(29,30)$.

ROS are predominantly generated from mitochondria and have a central role in cell death processes, including apoptosis and autophagy $(31,32)$. Under excessive oxidative stress, the accumulation of ROS reaches a threshold level that triggers opening of the MPTP or oxidation of the mitochondrial outer membrane, which in turn leads to the simultaneous collapse of mitochondrial membrane potential and a transient increase in ROS generation by the respiratory chain that transforms $\mathrm{O}_{2}$ into ATP $(33,34)$. Adpa-Mn was able to induce mitochondrial dysfunction, including mitochondrial membrane potential collapse, ROS accumulation and ATP depletion. Conversely, pretreatment with NAC and CSA significantly inhibited Adpa-Mn-induced ROS accumulation. In addition, NAC and CSA hampered Adpa-Mn-induced autophagy, whereas CsA decreased, but NAC aggravated, apoptosis (Fig. 5). CsA was also able to prevent Adpa-Mn-induced mitochondrial membrane potential collapse, whereas NAC could not. These data suggested that Adpa-Mn treatment destroyed mitochondria, thus leading to apoptosis of U251 cells; however, ROS that originated from the damaged mitochondria triggered protective autophagy.

In our previous study, the Adpa-Mn complex exhibited high toxicity on cancer cell lines, but showed weak DNA binding and cleavage activity (20). In addition, Adpa-Mn was shown to induce apoptotic cell death of HeLa cells (35). Conversely, whereas Adpa-Mn-induced autophagy in HeLa cells acted as a form of cell death, it was protective in U251 cells. These findings suggested that there may be different mechanisms underlying autophagy regulation in HeLa and U251 cells, which require further investigation.

In conclusion, the present study demonstrated that Adpa-Mn exhibited selective inhibition on glioma cell proliferation coupled with mitochondria-mediated apoptosis and increased autophagy. In addition, Adpa-Mn-induced autophagy exerted protective effects on glioma cells. Therefore, Adpa-Mn alone, or combined with autophagy inhibitors, may be considered a novel option for the treatment of glioma.

\section{Acknowledgements}

The authors of the present would you like to thank the following: The National Natural Science Foundation of China (grant no. 81402938); the Natural Science Foundation of Jiangsu Province (grant nos. BK2012710 and 20140575); and the Grant of Jiangsu University (grant no. 13JDG064).

\section{References}

1. Walker MD, Green SB, Byar DP, Alexander E Jr, Batzdorf U, Brooks WH, Hunt WE, MacCarty CS, Mahaley MS Jr, Mealey J Jr, et al: Randomized comparisons of radiotherapy and nitrosoureas for the treatment of malignant glioma after surgery. N Engl J Med 303: 1323-1329, 1980.

2. Subach BR, Witham TF, Kondziolka D, Lunsford LD, Bozik $M$ and Schiff D: Morbidity and survival after 1, 3-bis (2-chloroethyl)-1-nitrosourea wafer implantation for recurrent glioblastoma: A retrospective case-matched cohort series. Neurosurgery 45: 17-23, 1999.

3. Chamberlain MC and Kormanik P: Salvage chemotherapy with taxol for recurrent anaplastic astrocytomas. J Neurooncol 43 : 71-78, 1999.
4. Attardi G and Schatz G: Biogenesis of mitochondria. Annu Rev Cell Biol 4: 289-333, 1988.

5. Bender A, Opel D, Naumann I, Kappler R, Friedman L, von Schweinitz D, Debatin KM and Fulda S: PI3K inhibitors prime neuroblastoma cells for chemotherapy by shifting the balance towards pro-apoptotic Bcl-2 proteins and enhanced mitochondrial apoptosis. Oncogene 30: 494-503, 2011.

6. Susin SA, Lorenzo HK, Zamzami N, Marzo I, Snow BE, Brothers GM, Mangion J, Jacotot E, Costantini P, Loeffler M, et al: Molecular characterization of mitochondrial apoptosis-inducing factor. Nature 397: 441-446, 1999.

7. Nakahira K, Haspel JA, Rathinam VA, Lee SJ, Dolinay T, Lam HC, Englert JA, Rabinovitch M, Cernadas M, Kim HP, et al: Autophagy proteins regulate innate immune responses by inhibiting the release of mitochondrial DNA mediated by the NALP3 inflammasome. Nat Immunol 12: 222-230, 2011.

8. Mathew R and White E: Autophagy in tumorigenesis and energy metabolism: Friend by day, foe by night. Curr Opin Genet Dev 21: 113-119, 2011

9. He C and Klionsky DJ: Regulation mechanisms and signaling pathways of autophagy. Annu Rev Genet 43: 67-93, 2009.

10. Kimura T, Takabatake Y, Takahashi A and Isaka Y: Chloroquine in cancer therapy: A double-edged sword of autophagy. Cancer Rese 73: 3-7, 2013.

11. Williams R: Free manganese (II) and iron (II) cations can act as intracellular cell controls. FEBS Lett 140: 3-10, 1982.

12. Ansari KI, Kasiri S, Grant JD and Mandal SS: Apoptosis and anti-tumour activities of manganese(III)-salen and -salphen complexes. Dalton Trans: 8525-8531, 2009.

13. Kono Y and Fridovich I: Isolation and characterization of the pseudocatalase of Lactobacillus plantarum. J Biol Chem 258: 6015-6019, 1983

14. Law NA, Caudle T and Pecoraro VL: Manganese redox enzymes and model systems: Properties, structures and reactivity. Adv Inorg Chem 46: 305-440, 1998.

15. Luk E, Carroll M, Baker M and Culotta VC: Manganese activation of superoxide dismutase 2 in Saccharomyces cerevisiae requires MTM1, a member of the mitochondrial carrier family. Proc Natl Acad Sci USA 100: 10353-10357, 2003.

16. Calzolari A, Oliviero I, Deaglio S, Mariani G, Biffoni M, Sposi NM, Malavasi F, Peschle C and Testa U: Transferrin receptor 2 is frequently expressed in human cancer cell lines. Blood Cells Mol Dis 39: 82-91, 2007.

17. Aschner M and Aschner JL: Manganese transport across the blood-brain barrier: Relationship to iron homeostasis. Brain Res Bull 24: 857-860, 1990.

18. Aschner M and Gannon M: Manganese (Mn) transport across the rat blood-brain barrier: Saturable and transferrin-dependent transport mechanisms. Brain Res Bull 33: 345-349, 1994.

19. Li SJ, Jiang L, Fu X, Huang S, Huang YN, Li XR, Chen JW, Li Y, Luo HL, Wang F, et al: Pallidal index as biomarker of manganese brain accumulation and associated with manganese levels in blood: A meta-analysis. PloS One 9: e93900, 2014.

20. Chen QY, Huang J, Li JF and Gao J: Synthesis, interaction with mitochondrial and cancer cells of a dinuclear manganese(II) complex: $\mathrm{Mn}_{2}(\mathrm{Adpa})_{2} \mathrm{Cl}_{4}$. Chinese J Inorg Chem 24: 1789-1793, 2008 (In Chinese).

21. McCarthy KD and de Vellis J: Preparation of separate astroglial and oligodendroglial cell cultures from rat cerebral tissue. J Cell Biol 85: 890-902, 1980.

22. Lemasters JJ, Qian T, He L, Kim JS, Elmore SP, Cascio WE and Brenner DA: Role of mitochondrial inner membrane permeabilization in necrotic cell death, apoptosis, and autophagy. Antioxid Redox Signal 4: 769-781, 2002

23. Tanida I, Minematsu-Ikeguchi N, Ueno T and Kominami E: Lysosomal turnover, but not a cellular level, of endogenous LC3 is a marker for autophagy. Autophagy 1: 84-91, 2005.

24. Eisenberg-Lerner A, Bialik S, Simon HU and Kimchi A: Life and death partners: Apoptosis, autophagy and the cross-talk between them. Cell Death Differ 16: 966-975, 2009.

25. Desagher S and Martinou JC: Mitochondria as the central control point of apoptosis. Trends Cell Biol 10: 369-377, 2000.

26. Thorburn A: Autophagy and its effects: Making sense of double-edged swords. PLoS Biol 12: e1001967, 2014.

27. Klionsky DJ and Emr SD: Autophagy as a regulated pathway of cellular degradation. Science 290: 1717-1721, 2000.

28. Galluzzi L, Maiuri MC, Vitale I, Zischka H, Castedo M, Zitvogel L and Kroemer G: Cell death modalities: Classification and pathophysiological implications. Cell Death Differ 14: 1237-1243, 2007. 
29. Han J, Hou W, Goldstein LA, Lu C, Stolz DB, Yin XM and Rabinowich $\mathrm{H}$ : Involvement of protective autophagy in TRAIL resistance of apoptosis-defective tumor cells. J Biol Chem 283: 19665-19677, 2008.

30. Li J, Hou N, Faried A, Tsutsumi S, Takeuchi T and Kuwano H: Inhibition of autophagy by 3-MA enhances the effect of 5-FU-induced apoptosis in colon cancer cells. Ann Surg Oncol 16: 761-771, 2009.

31. Scherz-Shouval R, Shvets E, Fass E, Shorer H, Gil L and Elazar Z: Reactive oxygen species are essential for autophagy and specifically regulate the activity of Atg4. EMBO J 26: 1749-1760, 2007.
32. Scherz-Shouval R and Elazar Z: ROS, mitochondria and the regulation of autophagy. Trends Cell Biol 17: 422-427, 2007.

33. Garlid KD and Beavis AD: Evidence for the existence of an inner membrane anion channel in mitochondria. Biochim Biophys Acta 853: 187-204, 1986.

34. Zorov DB, Juhaszova $M$ and Sollott SJ: Mitochondrial ROS-induced ROS release: An update and review. Biochim Biophys Acta 1757: 509-517, 2006.

35. Liu J, Guo W,Li J,Li X, Geng J, Chen Q and Gao J: Tumor-targeting novel manganese complex induces ROS-mediated apoptotic and autophagic cancer cell death. Int J Mol Med 35: 607-616, 2015. 